\title{
Letter
}

\section{Subacute peripheral neuropathy of unknown cause complicating active borderline (dimorphous) leprosy}

Sir: Leprosy is uncommon in the United Kingdom and this is perhaps one reason why patients are referred to special departments, sometimes for months or years, before a diagnosis is established. ${ }^{1}$ Particularly in the case of those forms of the disease called borderline or dimorphous, which lie between the tuberculoid and lepromatous poles of the spectrum of leprosy, various factors, including infection, trauma, psychological upset, drugs and pregnancy may produce immunological disturbances which are damaging to the patient's tissues and which may contribute to difficulties in diagnosis. Furthermore, patients with leprosy may also suffer from other conditions causing neuropathy. We recently encountered a patient with leprosy in the UK whose clinical course was complicated by the development of a sub-acute peripheral neuropathy of unknown cause.

A 38-year-old female Iraqi schoolteacher first presented in 1965 with numbness in the right hand. Over the next 5 years she developed progressive weakness of both hands and feet and in 1968 a nerve biopsy and myelogram were performed in Baghdad, but no diagnosis was made. From 1970 to 1976 her hands and feet became increasingly deformed. At this stage a visiting neurologist made the diagnosis of leprosy and she was started on dapsone 200 $\mathrm{mg}$ daily which was reduced to $100 \mathrm{mg}$ and stopped when she became pregnant in 1977. In 1979, she re-started dapsone at $100 \mathrm{mg} /$ day but stopped after one year and took no further medication of any kind thereafter except vitamin B complex tablets.

Three months before arrival in the UK in 1981 , she developed an acute illness with sore thoat, fever, mild aches and pains all over the body, marked swelling of the hands and feet and the rapid development of bilateral wrist and foot drop. Her voice became weak and she had choking dysphagia with nasal regurgitation. She was taking no drugs apart from vitamin B complex and drank no alcohol. These symptoms had improved but she still remained weak. Examination after arrival in the UK revealed a thin apprehensive woman of above average intelligence with widespread wasting of the limb muscles. Under her right breast there was a 2 by $3 \mathrm{~cm}$ raised, red lesion with indefinite outer borders, but a definite "punched out" inner border. There were two hypopigmented macules on the back of the neck, of vague outline and each about $2 \mathrm{~cm}$ in diameter. In the cranial nerves she had decreased touch and pain perception on the left side of her face. There was bilateral facial nerve weakness of lower motor neuron type, much worse on the left side and inability to close the lower lids of her eyes (lagophthalmos). Corneal reflexes were intact. There was asymmetrical thickening of many peripheral nerves, including the great auricular, supra-clavicular, lateral popliteal and superficial peroneal. She had gross wrist and foot drop with a marked flexion deformity of her hands; there was resorption of the distal phalanges shown on radiographs but no other trophic lesions. Limbs showed severe distal weakness with normal tone. She had symmetrical gloveand-stocking sensory loss to above her knees and elbows, whilst vibration sense was retained in her feet but not in her hands. There were ill-defined patches of sensory loss over the abdomen and between the scapulae. All peripheral reflexes were absent and she was unable to walk. Investigations revealed $\mathrm{Hb} 13.4 \mathrm{~g} / \mathrm{dl}$, WBC $8.9 \times 10^{9} / 1$, platelets $447 \times 10^{9} / 1$, ESR 33 $\mathrm{mm}$ in the first hour. Urea and electrolytes, liver function tests and glucose were normal. Serum B12, folate, glucose-6phosphate dehydrogenase, faecal and urinary porphyrins, serum lead and mercury levels were all normal. Serology for syphilis (RPR and TPHA) was negative. The reciprocal viral titres (complement fixation) were as follows: Herpes simplex 16 , varicella zoster 8, enterovirus 8 and CMV 64 . Although the CMV IgM was positive, the latex test for rheumatoid factor was positive but the Rose Waaler test was negative indicating a possible false-positive tes: for CMV infection. IgM to EBV was negative; the T3 was $2 \cdot 3 \mathrm{nmol} / \mathrm{l}$. The immunoglobulins were all raised, IgG $25 \cdot 7 \mathrm{~g} / 1$ (normal $7 \cdot 2-19) ;$ IgA $5 \cdot 3$ g/l (0.8-5); IgM $2 \cdot 1$ g/l $(0 \cdot 5-2)$. Auto-antibodies including antinuclear factor, gastric parietal cell antibody, smooth muscle antibody and antimitochondrial antibody were all negative. The throat swab grew a light growth of beta haemolytic streptococci group A but no corynebacteria. The Schick test was negative. An initial diphtheria anti-toxin level was $0 \cdot 2-1 \mathrm{u} / \mathrm{ml}$ and one month later it was 1-2 $\mathrm{u} / \mathrm{ml}$. (This indicated previous exposure as she had not been immunised). A lumbar puncture on first admission was traumatic; it showed no white cells, red cells $1,600 \times 10^{9} / 1$, normal glucose, protein $\bar{Z}$ $1.7 \mathrm{~g} / \mathrm{l}$ and no organisms. Repeat lumbar puncture 18 days later confirmed a raised protein of $2.0 \mathrm{~g} / \mathrm{l}$, with 8 white cells (all lymphocytes) red cells $106 \times 10^{9} / 1$; the $\overline{\bar{c}}$ albumin was $1.1 \mathrm{~g} / \mathrm{l}$ and $\mathrm{IgG} 0.38 \mathrm{~g} / \mathrm{l}$ (ratio $\square$ $0 \cdot 39)$. There were no oligoclonal bands. Electromyographic studies (Dr Geoffrey $\stackrel{\bigcirc}{\supset}$ Rushworth, Oxford): using coaxial needle electrodes, the following muscles were examined on the left side of the bodybrachio-radialis, biceps, deltoid, abductor $\stackrel{\bar{F}}{+}$ pollicis brevis, first dorsal interosseus, $\bar{O}$ abductor digiti minimi, frontalis, levator $\frac{\bar{O}}{\sigma}$ labii superioris and mentalis. The conclu- $\frac{\bar{\sigma}}{\bar{D}}$ sions were as follows "The small muscles of $\frac{\vec{D}}{\mathbb{D}}$

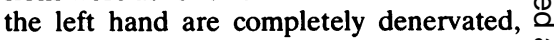
and it would appear that maximum de- $\omega$ generation of their motor fibres has taken $\overrightarrow{0}$ place. The left brachial radialis is severely ? denervated and surviving motor nerve $\overrightarrow{\vec{\omega}}$ fibres conduct at a reduced velocity (estimated at $25 \mathrm{~m} / \mathrm{s}$ ). The left biceps is also moderately severely denervated, and again $\frac{2}{2}$ there is some reduction in the motor fibres innervating this muscle. The deltoid is ? much less affected. The left facial muscles $\vec{\square}$ are partly denervated and again the survivo $\vec{\partial}$ ing motor nerve fibres show a marke 엉 reduction in their maximum motor ve $\infty$ ocities. Mentalis is the least affected of the facial muscles. These findings indicate tha $\rightarrow$ segmental demyelination of motor nere $Z$ fibres plays some part in the pathologicad process and that it affects principally the proximal muscles." The complete denerva. tion of the distal muscles was considered be due to leprosy. Although the involve $\vec{e}$ ment of the proximal muscles in a recent $\stackrel{\infty}{\omega}$ episode could have been due to a Guillain-Barré syndrome, it was pointed out that the onset had been sub-acute and that there has been some axonal degenera- $\frac{\partial}{0}$ tion as well as segmental demyelinafion. ECG was normal. Radiographs of the skull $\stackrel{\circ}{\triangleright}$ revealed evidence of her myelogram $13 \stackrel{2}{\rightarrow}$ years earlier with opacities at the base of $\overrightarrow{\overline{0}}$ the skull due to contrast medium. Slit-skin 3 smears of her skin lesions were negative for acid-fast bacilli, but bacilli were found in granular or fragmented form in biopsies of the left great auricular nerve and of the skin lesion below the right breast. These? biopsies also showed a picture of mid- $\frac{5}{3}$ borderline (dimorphous) leprosy (BB on the Ridley-Jopling scale ${ }^{2}$ ), with features of $\delta$ reversal (upgrading) reaction. ${ }^{3}$ She was treated with rifampicin, dapsone and pre-을 dnisolone and improved markedly over 2 months. On discharge, she was able to walk. A cerebrospinal fluid protein discharge was $0.7 \mathrm{~g} / \mathrm{l}$. 
The main diagnosis of boderline leprosy in reversal (upgrading) reaction in this woman was undisputed. There was no evidence to suggest an excessive intake of dapsone, or the ingestion (in normal or excessive doses) of any drug which could have caused a neuropathy, and the clinical findings were not in keeping with heavy metal poisoning. Rapid deterioration of nerve function due to cell-mediated immune processes in a reversal (upgrading) reaction in leprosy was a possibility, but this could not have accounted for the raised CSF protein, regurgitation of fluid through the nose and the total loss of limb jerks. None of the other conditions considered and investigated offered any explanation for such extensive and severe neuropathy of sudden onset. The crucial question of her clinical progress between the acute illness in February and her presentation in this country in early May cannot be fully answered, partly because of language difficulties. However, the available evidence obtained through interpreters suggested that her paralysis progressed over a period of at least 2 months. Thus, although a Guillain-Barré syndrome was considered, it is perhaps more likely that the complicating illness was a sub-acute peripheral neuropathy of unknown cause.

AC MCDOUGALL

The Dermatology Department, Slade Hospital, Oxford, $\mathrm{OX} 3 \mathrm{7JH}$ G PASVOL

Tropical Medicine Unit, the Nuffield Department of Clinical Medicine, John Radcliffe Hospital, Oxford, $O X 39 D U$

\section{References}

' Powell S, McDougall AC. Clinical recognition of leprosy; some factors leading to delays in diagnosis. Br Med J 1974;612-13.

${ }^{2}$ Ridley DS, Jopling WH. The classification of leprosy according to immunity. Int J Lepr 1966;34:255-73.

${ }^{3}$ Ridley DS. Reactions in leprosy. Lep Rev 1969;40:77-81.

Accepted 20 June 1983 